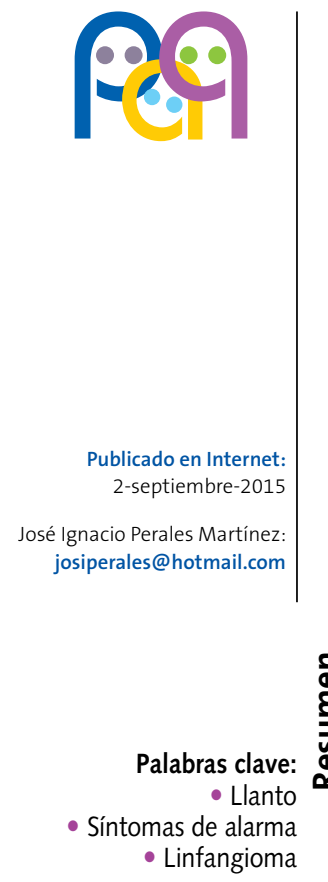

\title{
Llanto al mes de vida. No todo son cólicos
}

\section{J. I. Perales Martínez ${ }^{a}$, B. Pina Marqués ${ }^{b}$, S. Congost Marín ${ }^{a}$, M. Odriozola Grijalba ${ }^{a}$, M. Vara Callau ${ }^{a}$, P. Lalaguna Mallada ${ }^{a}$}

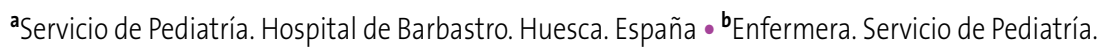
Hospital Universitario Miguel Servet. Zaragoza. España.

\section{Crying in a one-month-old baby. Not all are crampings}

El llanto de los lactantes es uno de los motivos de consulta más frecuentes en las Urgencias Pediátricas. La mayoría de las ocasiones son cuadros autolimitados, pero hay que prestar especial atención a los signos y síntomas de alarma para realizar otras pruebas complementarias. Presentamos el caso de un lactante de un mes de vida que asociaba distensión abdominal y rechazo de las tomas. En las pruebas de imagen se apreció una masa multiquística en abdomen. La laparotomía evidenció una malformación linfática en el íleon medio, con confirmación anatomopatológica de linfagioma quístico mesentérico.

\section{INTRODUCCIÓN}

La irritabilidad, o con mayor frecuencia el llanto, no es solamente una forma de expresar dolor o malestar por parte del niño. En los niños más pequeños, es en ocasiones la única forma de comunicarse con sus padres ${ }^{1}$.

El llanto como tal es uno de los motivos de consulta más frecuentes de los niños de edad inferior a tres meses en un Servicio de Urgencias Pediátricas. En la mayoría de situaciones, el lactante se ha calmado en el trayecto desde el domicilio hasta el hospital, presentando en Urgencias un aspecto saludable. Cuando Ilora un bebé habitualmente lo hace porque está insatisfecho, por hambre, pañal húmedo o con deposición, demasiado arropamiento, sensación de frío, necesidad de contacto con la madre, etc. Lo más extendido y más habitual es la idea de que "un niño llora cuando tiene hambre"; esto, en ocasiones, no es correcto. Además, según va creciendo el niño, los padres aprenden a distinguir la llamada del niño cuando tiene hambre 
y que generalmente se expresa por medio de llanto. Hay que recordar que, en los niños pequeños, el llanto o irritabilidad puede ser la manifestación inicial de que un niño tiene fiebre, por eso es recomendable, en estos niños, tomar la temperatura rectal².

El lactante irritable o con llanto es una de las consultas más estresantes en las Urgencias Pediátricas. A medida que descendemos en la edad del paciente, más frecuente es que el llanto se presente como único punto de referencia en la anamnesis recogida a la familia. Por eso será de especial importancia una cuidadosa exploración física orientada a descartar patologías en las que un diagnóstico precoz mejora en gran manera el pronóstico. Interrogaremos siempre por las siguientes cuestiones: temperatura, comportamiento alimentario, vómitos, deposiciones, síntomas vegetativos y tipo de llanto (continuo/intermitente, consolable, horario, etc.). Suele ser raro que el llanto sea la expresión única de una enfermedad, habitualmente se añaden otros signos y síntomas. En el caso del lactante más joven es tranquilizador el hecho de que el niño se haya calmado en el trayecto hacia Urgencias, tome sin problemas o haya presentado episodios similares los días o semanas previos. Pero antes de etiquetar a este lactante de cólicos, será fundamental descartar las situaciones de riesgo y los signos de alarma que nos obligarán a un estudio más minucioso, como la presencia de fiebre, rechazo de las tomas, palpación abdominal patológica, aparición de vómitos o rectorragia, alteraciones de la fontanela anterior y el llanto inconsolable ${ }^{2}$.

\section{CASO CLÍNICO}

Lactante varón de un mes de vida, sin antecedentes personales de interés, que acude por tercera vez a Urgencias en la última semana con un cuadro de llanto parcialmente consolable. Había sido diagnosticado de cólicos del lactante y se recomendaron las medidas habituales. Toma lactancia materna exclusiva. En las últimas 48 horas realiza peor las tomas, el llanto, que inicialmente era vespertino, se mantiene todo el día y la madre no consigue calmarlo en brazos desde ayer. Se mantiene afebril y con excelente estado general, presenta adecuada ganancia ponderal, actualmente con un peso de $4 \mathrm{~kg}$. La exploración física impresiona de distensión abdominal y se palpa una masa a nivel periumbilical que se extiende hacia flanco derecho, sin apreciarse defensa evidente. Rechaza una toma de lactancia materna en Urgencias de forma franca y presenta un vómito alimenticio.

Con sospecha de obstrucción intestinal, se realiza una radiografía de abdomen (Fig. 1) en la que aparece un marcado silencio abdominal (ausencia de gas) en hemiabdomen derecho y efecto masa con desplazamiento de las asas intestinales hacia hemiabdomen izquierdo. La ecografía muestra a nivel centroabdominal una imagen de aproximadamente $9 \times 8,7 \times 7,9 \mathrm{~cm}$, que se comporta como gran lesión focal ocupante de espacio de características quísticas sin flujo vascular al Doppler color y multiloculada y multitabicada.

Se decide traslado a un centro de referencia para cirugía pediátrica. Se completa el estudio con resonancia

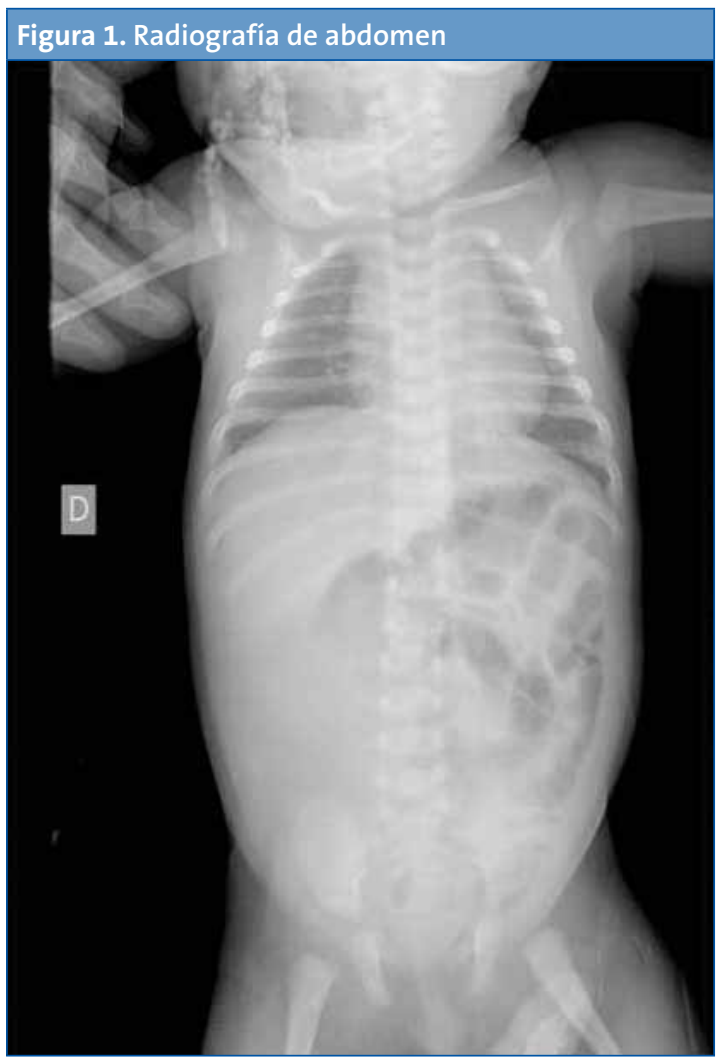




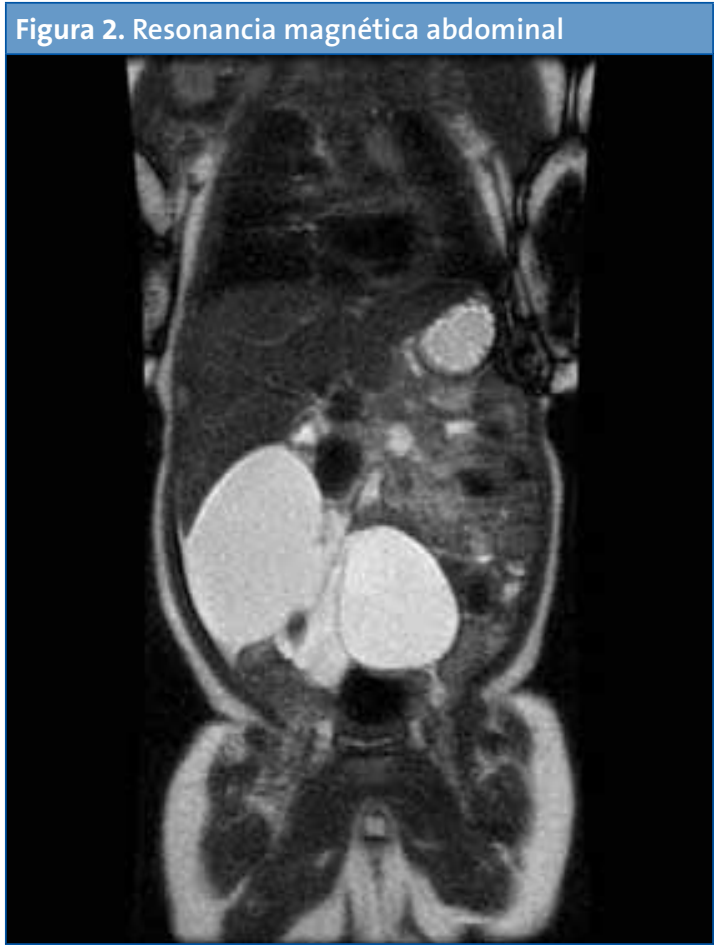

magnética (RM) de abdomen (Fig. 2), en la que aparece una voluminosa masa quística intraabdominal de unos $90 \times 60 \times 65 \mathrm{~mm}$, localizada principalmente en el flanco derecho y la región centroabdominal. Se extiende desde el borde inferior hepático, por delante del riñón derecho desplazando hacia la izquierda las estructuras intestinales. Se trata de una masa multilocular, de bordes bien definidos y paredes finas, con múltiples componentes quísticos de diferentes tamaños. La primera sospecha diagnóstica en un linfangioma quístico de mesenterio.

Es intervenido mediante laparotomía media y se procede a exéresis de una masa multiquística con contenido de líquido blanquecino que depende del mesenterio de un asa del íleon medio. Se reseca dicha asa y se realiza anastomosis término-terminal. El posoperatorio, las primeras 24 horas en Unidad de Cuidados Intensivos Pediátricos (UCIP) y el resto en planta, ha cursado sin incidencias y en ocho días se remite a su domicilio con adecuada tolerancia enteral y sin complicaciones.

La anatomía patológica confirma el diagnóstico de linfagioma quístico mesentérico al observar una masa quística multiseptada de aproximadamente $5 \mathrm{~cm}$ de diámetro con pared lisa blanquecina de un espesor de $0,1 \mathrm{~cm}$.

\section{DISCUSIÓN}

Los linfangiomas son un grupo poco frecuente (6\%) de neoplasias benignas, caracterizadas por malformaciones vasculares linfáticas. Son tumores propios de la edad pediátrica y la localización más frecuente es en el cuello y las axilas, en un 95\% de los $\operatorname{casos}^{3}$.

La etiología del linfangioma es desconocida, siendo la teoría más aceptada la congénita, debido a una falta de comunicación entre los vasos linfáticos durante la vida fetal de forma que los vasos ciegos se dilatan hasta formar un tumor quístico ${ }^{4}$. A nivel cervical podría apreciarse una anomalía del sistema linfático por la obstrucción del drenaje de los sacos linfáticos cervicales al sistema venoso yugular. El aumento de presión en el sistema linfático podría superar una obstrucción incompleta, lo que explicaría los casos de resolución espontánea ${ }^{5}$. También se ha postulado que pudieran guardar relación con traumatismos, cirugía previa o radioterapia ${ }^{6}$.

Morfológicamente se clasifican en tres tipos: linfangioma simple, formado por pequeños vasos linfáticos de paredes delgadas, linfangioma cavernoso constituido por vasos linfáticos de pequeño tamaño con capas conectivas de grosor irregular entre ellos, y linfangiomas o higroma quístico cuando está compuesto por grandes espacios linfáticos macroscópicos que poseen revestimiento de colágeno y músculo liso. Estos últimos, como el caso de nuestro paciente, tienen gran interés clínico porque su crecimiento puede comprometer el órgano donde asienta.

Los linfangiomas abdominales son raros y representan del 2-5\% del total, siendo en lugar de presentación más habitual el mesenterio $(45 \%)^{3}$. Se caracterizan por ser lesiones quísticas en "racimo de uvas" y multiloculadas, generalmente con contenido lechoso y/o hemorrágico. Histológicamente 
presentan un epitelio endotelial plano y tejido linfoide con espacios linfáticos dilatados, músculo liso y desarrollo hemangiomatoso, de ahí que muchos autores los consideren como hamartomas vasculares o como disembrioplasias del sistema linfático ${ }^{7}$.

El cuadro clínico de los linfangiomas abdominales es variable. Muchas veces son asintomáticos, por lo que el hallazgo es casual, debido a un crecimiento lento. Pero en otras ocasiones aparece distensión abdominal, masa palpable e incluso signos de abdomen agudo u obstrucción intestinal debido a la compresión por efecto masa. También se ha reportado la presencia de sangrado debido a torsión o erosión del quiste, lo cual también ocasiona sintomatología.

Son fundamentales para el diagnóstico las pruebas de imagen: ecografía, tomografía computarizada (TC) y RM. Se pueden detectar también por ecografía fetal. El diagnóstico definitivo lo aporta la exéresis quirúrgica y la anatomía patológica posterior.

El diagnóstico diferencial se realiza con otros tumores quísticos retroperitoneales prevalentes en edad pediátrica, como teratoma quístico benigno, quistes de mesenterio y otras masas retroperitoneales ${ }^{8}$.

El tratamiento de elección de los linfangiomas abdominales es la resección quirúrgica completa. Debe llevarse a cabo tan pronto sea posible, a menos que exista evidencia de infección que debería posponer la cirugía tres meses. Otras terapias, como el láser, la aspiración, drenaje e irrigación del linfangioma intraabdominal, dan pobres resultados. La inyección intraquística de adhesivo de fibrina, ciclofosfamida intravenosa y la escleroterapia con bleomicina y el OK-432 dan recidivas en hasta un $10-15 \%{ }^{9}$ de los casos. En cuanto al comportamiento, aunque su naturaleza es benigna, puede ser localmente invasivo por lo que es recomendable la resección completa del tejido adyacente. Si la resección no fuera posible se considera la marsupialización como alternativa aceptable. En un estudio de 145 linfangiomas en edad pediátrica objetivaron que las complicaciones quirúrgicas aparecían en relación al tamaño y la localización, siendo más frecuentes en aquellos de gran tamaño y con asiento en el mesenterio ${ }^{10}$. A su vez comprobaron un $6 \%$ de recidivas posiblemente secundarias a resección quirúrgica incompleta, con permanencia de canales linfáticos residuales en linfangiomas con componente cavernoso ${ }^{10}$.

Como conclusiones finales, recordar la gran importancia que ha tenido en nuestro paciente prestar atención a los signos de alarma y no etiquetar de forma inicial, porque no siempre lo más frecuente es lo que aparece. En este caso podría haberse diagnosticado el llanto como unos cólicos pero la distensión abdominal y el rechazo de las tomas nos apoyaron para solicitar una prueba de imagen que nos ayudó al diagnóstico definitivo. Nuestro objetivo es, por lo tanto, destacar la importancia de la anamnesis y la exploración física en pediatría que nos orientarán hacia el diagnóstico en la mayoría de las situaciones.

\section{CONFLICTO DE INTERESES}

Los autores declaran no presentar conflictos de intereses en relación con la preparación y publicación de este artículo.

\section{ABREVIATURAS}

RM: resonancia magnética • TC: tomografía computarizada - UCIP: unidad de cuidados intensivos pediátricos. 


\section{BIBLIOGRAFİA}

1. Barr RG, Rotman A, Yaremko J, Leduc D, Francoeur E. The crying of infants with colic: a controlled empirical description. Pediatrics. 1992;90:14-21.

2. Mintegi S. Llanto. Irritabilidad. En: Benito J, Mintegi S. Diagnóstico y tratamiento de Urgencias Pediátricas. 3. a edición. Madrid: Ergón; 2002. p.478-80.

3. Alvite Canosa M, Alonso Fernández L, Seoane Vigo M, Pérez Grobas J, Berdeal Díaz M, Bouzón Alejandro A. Linfangioma abdominal en una adolescente. Rev Esp Enferm Digest. 2008;100:515-22.

4. Fontirroche Cruz R, González Dalmau L, Barroetabeña Riol Y, Araujo Mejías M. Linfangioma quístico abdominal. A propósito de dos casos pediátricos. Mediciego. 2010;16

5. Tanriverdi HA, Ertan AK, Hendrik HJ, Remberger K, Schmidt W. Outcome of cystic hygroma in fetuses with normal karyotypes depends on associated findings. Eur J Obstet Gynecol ReprodBiol. 2005;118:40-6.

6. Reyes I, Rossel G, Pacheco A. Parada X, Casanova ME. Linfangioma de intestino delgado. Rev Chil Cir. 2004; 56:66-70.

7. Steyaert H, Guiitard J. Abdominal cystic limphangioma in children: benign lesions that can have a proliferative course. J Pediatr Surg. 2006;31:677-80

8. De Vries JJ, Vogten JM, de Bruin PC, Boerma D, Van de Pavoordt HD, Hagendoorn J. Mesenterical lymphangiomatosis causing volvulus and intestinal obstruction. Lymphat Res Biol. 2007;5:269-73.

9. Perrot $M$, Rostan O. Abdominal lymphangioma in adults and children. Br J Surg. 1998;85:395-7.

10. Gimeno M, Colomar P, González I, ollero JM. Aspectos clínicos y morfológicos de los linfangiomas infantiles: Revisión de 145 casos. An Esp Pediatr. 1996;45:25-8. 